\title{
Latest Results in Charmed Baryons Spectroscopy
}

\author{
Elena Solovieva ${ }^{1, *}$ \\ ${ }^{1}$ P.N. Lebedev Physical Institute of the Russian Academy of Sciences, 53 Leninskiy Prospekt, Moscow, \\ 119991, Russia
}

\begin{abstract}
An overview of recent developments in the spectroscopy of charmed baryons is given. The classification of charmed baryons is presented, a quark model for ground states is briefly described, and the energy levels of excited states are analysed.
\end{abstract}

\section{Introduction}

The spectroscopy of charmed baryons is complex and beautiful. The presence of three quarks provides numerous degrees of freedom, which leads to a much larger number of states than for the charmed mesons. At the same time, the big difference between the masses of the heavy $c$-quark and light quarks provides a natural way to classify and understand these states: Heavy Quark Effective Theory (HQET). The spectrum of known states with one charmed quark can be divided into three broad areas: ground states, which confirm the constituent quark model; low-lying excited states that are well described by HQET; higher excitations for which the situation is more complicated.

\section{Quark Model for Ground States}

In the constituent quark model [1] baryons consisting of $u$-, $d-, s-, c$-quarks can be systematized into $S U(4)$ multiplets in accordance with symmetry of flavour, spin and spatial wave functions. All states in each individual $S U(4)$ multiplet have the same total angular momentum $J$ and parity $P$, but can have different quark flavours. For excited states with several units of the orbital angular momentum, the number of possible multiplets becomes large, but for the ground states the picture is much simpler. This scheme is not completely accurate, since different states with the same conserved quantum numbers will mix, but it works great for ground states.

Quarks are fermions, so the total wave function of the baryon should be antisymmetric when the quarks are swapped (though strictly speaking, it should be antisymmetric only when swapping quarks with the same mass, but in order to build a model, $S U(4)$ is considered to be exact symmetry). Baryons - colour singlets, therefore, have an antisymmetric colour wave function. For the ground state the orbital angular momentum is $L=0$ ( $S$-wave) and the spatial wave function are symmetric. Thus, the product of the wave functions of the spin and flavour for the ground state baryons should also be symmetric. This can be achieved in

*e-mail: lena@lebedev.ru 

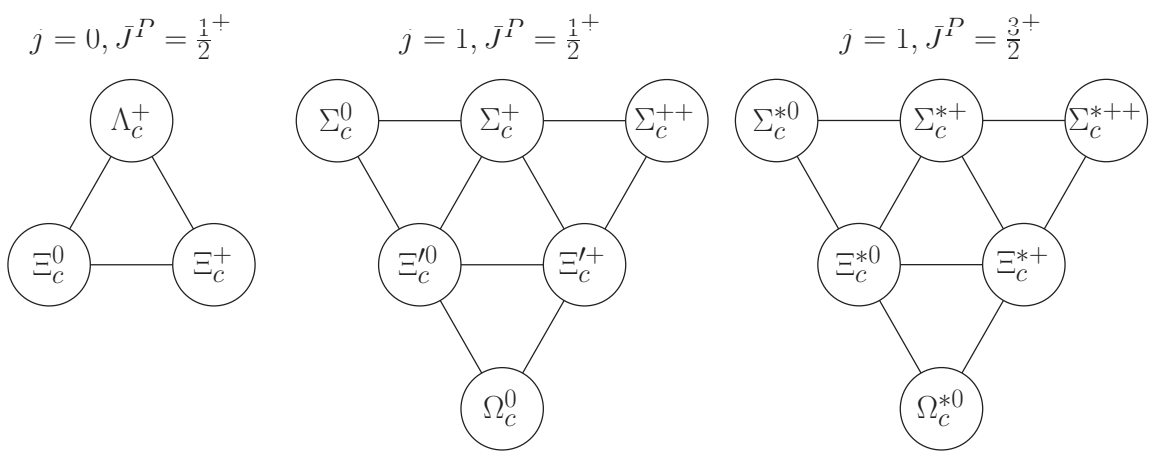

Figure 1. $S U(3)$ multiplets that contain the ground states of charmed baryons, grouped according to the total angular momentum $j$ of the light diquark and the angular parity $J^{P}$ of baryon.

two ways: both wave functions can be completely symmetric, or have mixed symmetry with a symmetric product.

One can consider baryons with $C=1$ as consisting of a heavy $c$-quark and a light diquark with quantum numbers $j^{p}$, where $j$ is the total angular momentum and $p$ is the spatial parity of the diquark. Assuming isospin symmetry and designating $u$ - or $d$-quarks as $q_{l}$, there are four possibilities for the structure of the diquark:

- $q q$ with isospin 0 (flavour antisymmetric);

- $q q$ with isospin 1 (flavour symmetric);

- $s q$ with isospin $1 / 2$ (either);

- $s s$ with isospin 0 (flavour symmetric).

They correspond to the states of $\Lambda_{c^{-}}, \Sigma_{c^{-}}, \Xi_{c^{-}}$, and $\Omega_{c^{-}}$-baryons respectively.

The wave function of the diquark should be antisymmetric when the quarks are swapped. Its colour wave function is antisymmetric, and in the ground state the spatial wave function is symmetric, therefore, it can be either symmetric in spin and flavour $\left(j^{p}=1^{+}\right)$, or antisymmetric in spin and flavour $\left(j^{p}=0^{+}\right)$. The combination of a diquark with a heavy $c$-quark determines the possible states illustrated in figure 1, where the multiplets of the full $S U(3)$ symmetry (formed by the $u^{-}, d$-, and $s$-quarks) are shown. All states with $J^{P}=\frac{1}{2}^{+}$are members of the same multiplet as the proton; while states with $J^{P}=\frac{3}{2}^{+}$are members of the same multiplet as $\Delta$ and $\Omega$ (figure 2). There is a second isospin doublet of states $\Xi_{c}$ with $J^{P}=\frac{1}{2}^{+}$, denoted as $\Xi_{c}^{\prime}$.

The constituent quark model predicts the relationships between the masses of states, their existence and quantum numbers. Though it should be noted that in many cases the total angular momentum and parity of the state are assigned based on the predictions of the quark model, and not measurements. Therefore, at present, the experimental determination of $J^{P}$ is one of the main problems in the spectroscopy of charmed baryons.

The quark model also predicts baryons with two charm quarks and one light quark, as shown in figure 2. Fewer distinct configurations are possible than for the $C=1$ baryons, because flavours of the two quarks are identical. Three weakly-decaying ground states with $J^{P}=\frac{1}{2}^{+}\left(\Xi_{c c}^{+}, \Xi_{c c}^{++}, \Omega_{c c}^{+}\right)$and three more states with $J^{P}=\frac{3}{2}^{+}\left(\Xi_{c c}^{*+}, \Xi_{c c}^{*+}, \Omega_{c c}^{*+}\right)$ are expected. 


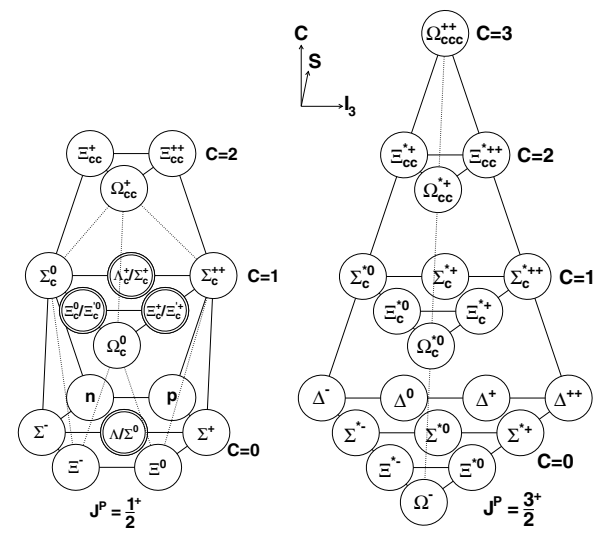

Figure 2. $S U(4)$ multiplets that contain the ground states of baryons placed according angular parity $\left(J^{P}\right)$, projections of isospin $\left(I_{3}\right)$, strangeness $(S)$ and charm $(C)$. Double ringed points mean that two states have the same quantum numbers $J^{P}, I_{3}, S$ and $C$, but different total angular momentum of easy diquark.

\section{Excited States}

Baryons can be given orbital $(l)$ or radial $(k)$ excitations. In each case there are two degrees of freedom (denoted by $\rho, \lambda$ ), because it is a three-particle system. For baryons with one heavy quark (with mass $M$ ) and two light quarks (with mass $m$ ), the natural way to describe them is to divide the system into a light diquark and a heavy quark. Taking a simple potential model based on a harmonic oscillator, one can obtain energy levels [2]:

$$
E=\sqrt{\frac{K}{m}}\left(3+2 l_{\rho}+4 k_{\rho}\right)+\sqrt{\frac{K}{\mu}}\left(3+2 l_{\lambda}+4 k_{\lambda}\right),
$$

where $l_{\rho, \lambda}=0,1,2, \ldots ; k_{\rho, \lambda}=0,1,2, \ldots ; K$ is a constant describing the potential; and $\mu=$ $\left(\frac{2}{3 M}+\frac{1}{3 m}\right)^{-1} \approx 3 m$ in the limit of a heavy $c$-quark. Thus, the $\rho$ excitations (inside the diquark) require approximately three times more energy than the corresponding $\lambda$ excitation (between the quark and the diquark). Therefore the lowest excitations are states with $l_{\lambda}=1$ and the remaining quantum numbers being null, i.e. $L=1$. Inside this band there will be additional splitting, for example, due to spin-spin and spin-orbit interactions. The second strip will consist of two groups of states having comparable energies: with $l_{\lambda}=2(L=2)$ and with $k_{\lambda}=1(L=0)$ with the remaining null quantum numbers. Above the second band degeneracy grows further, and it is precisely in this region that experimental data are lacking.

Having said all this, it is important to keep in mind that the states with all conserved external quantum numbers $(J, P, I, C, S)$ being the same can mix. Therefore one should be very careful when interpreting the observed resonances as certain expected states, especially for high excitations.

\section{$4 \Lambda_{c}$ Family}

The table 1 gives a brief summary of the experimentally excited states of the observed $\Lambda_{c}^{+}$baryons. The first two states, $\Lambda_{c}(2595)^{+}$and $\Lambda_{c}(2625)^{+}$, are well studied. Based on the 
Table 1. A brief summary of the excited states of the $\Lambda_{c}^{+}$-baryons [3].

\begin{tabular}{|c|c|c|c|c|}
\hline State & Decay mode & Mass, MeV/c & Width, MeV & $J^{P}$ \\
\hline \hline$\Lambda_{c}(2595)^{+}$ & $\Lambda_{c}^{+} \pi^{+} \pi^{-}, \Sigma_{c} \pi$ & $2592.3 \pm 0.3$ & $2.6 \pm 0.6$ & $\frac{1}{2}^{-}$ \\
\hline$\Lambda_{c}(2625)^{+}$ & $\Lambda_{c}^{+} \pi^{+} \pi^{-}, \Sigma_{c} \pi$ & $2628.11 \pm 0.19$ & $<0.97 @ 90 \% \mathrm{CL}$ & $\frac{3}{2}^{-}$ \\
\hline$\Lambda_{c}(2765)^{+}$ & $\Lambda_{c}^{+} \pi^{+} \pi^{-}, \Sigma_{c} \pi$ & $2766.6 \pm 2.4$ & $\sim 50$ & \\
\hline$\Lambda_{c}(2860)^{+}$ & $D^{0} p$, & $2856.1_{-6.0}^{+2.3}$ & $68_{-22}^{+12}$ & $\frac{3}{2}^{+}$ \\
\hline$\Lambda_{c}(2880)^{+}$ & $\Lambda_{c}^{+} \pi^{+} \pi^{-}, \Sigma_{c} \pi$, & $2881.6 \pm 0.2$ & $5.6_{-0.6}^{+0.8}$ & $\frac{5}{2}^{+}$ \\
& $\Sigma_{c}(2520) \pi, D^{0} p$ & & & \\
\hline$\Lambda_{c}(2940)^{+}$ & $D^{0} p, \Sigma_{c} \pi$ & $2939.3_{-1.5}^{+1.3}$ & $20_{-5}^{+6}$ & \\
\hline
\end{tabular}

measured masses, it is believed that they are orbital excitations of $\Lambda_{c}^{+}$with the total moment of light quarks $j=1$. Therefore, they were assigned the quantum numbers $J^{P}=\left(\frac{1}{2}\right)^{-}$and $J^{P}=\left(\frac{3}{2}\right)^{-}$. Two of the following states, $\Lambda_{c}(2765)^{+}$and $\Lambda_{c}(2880)^{+}$, were discovered by the CLEO collaboration in the channel $\Lambda_{c}^{+} \pi^{+} \pi^{-}$[4]. It also turned out that they can also decay to $\Sigma_{c}(2445)^{++/ 0} \pi^{-/+}$final state [5].

The experimental status of $\Lambda_{c}(2765)^{+}$is very poor: its spin, parity and even isospin are not determined yet and CLEO did not determine the uncertainty on its width. The nature of $\Lambda_{c}(2765)^{+}$is rather controversial and experimental determination of its quantum numbers is long overdue. Belle performed a determination of the isospin of $\Lambda_{c}(2765)^{+}$by searching for possible isospin partners of the state in the decay modes to $\Sigma_{c}(2455)^{++/ 0} \pi^{0}$ and further $\rightarrow \Lambda_{c} \pi^{ \pm} \pi^{0}$ [6]. The isospin is determined to be $I=0$, therefore the state is indeed $\Lambda_{c}$. The result is rather inconsistent with the latest quark model calculation, but not with the older ones. In addition, some theoretical predictions are excluded by this. However, in order to discuss in more detail the nature of this state it is highly desirable to have the experimental determination of its other quantum numbers (i.e. spin and parity).

Later, the BaBar experiment announced that $\Lambda_{c}(2880)^{+}$state has the mode $D^{0} p$ [7], which was the first example of the decay of a charmed baryon into a charmed meson and light baryon. In the same analysis, another state that decays into $D^{0} p-\Lambda_{c}(2940)^{+}-$was discovered. Since no signal indications were found when examining the final state of $D^{+} p$, it was concluded that $\Lambda_{c}(2880)^{+}$and $\Lambda_{c}(2940)^{+}$are indeed excited states of $\operatorname{Lambda}_{c}^{+}$, not of $\Sigma_{c}$. The Belle experiment performed an angular analysis, the results of which spoke in favour of the quantum number $\frac{5}{2}$ for the total angular momentum $\Lambda_{c}(2880)^{+}$[5]. In addition, the measured decay probability ratio $\mathcal{B}\left(\Lambda_{c}(2880)^{+} \rightarrow \Sigma_{c}(2520) \pi^{ \pm}\right) / \mathcal{B}\left(\Lambda_{c}(2880)^{+} \rightarrow \Sigma_{c}(2455) \pi^{ \pm}\right)=$ $(0.225 \pm 0.062 \pm 0.025)$, combined with theoretical predictions based on the symmetry of heavy quarks [8], indicated positive parity.

Recently LHCb Collaboration performed an amplitude analysis of the Cabibbofavoured decay $\Lambda_{b}^{0} \rightarrow D^{0} p \pi^{-}$, including resonant contributions in the $D^{0} p$ channel associated with intermediate excited $\Lambda_{c}$ states [9]. Among other things this work provided important information on the spectroscopy of excited $\Lambda_{c}$ states. It was confirmed that the preferred spin of the $\Lambda_{c}(2880)^{+}$state is $J=5 / 2$, with the $J=7 / 2$ hypothesis disfavoured by $4.0 \sigma$ (standard deviations). Solutions with $J=$ $1 / 2$ and $3 / 2$ were excluded with a significance of more than $5 \sigma$. The mass and width of the $\Lambda_{c}(2880)^{+}$state were measured with slightly better accuracy and turned out to be: $M\left(\Lambda_{c}(2880)^{+}\right)=\left[2881.75 \pm 0.29\right.$ (stat) \pm 0.07 (syst) ${ }_{-0.20}^{+0.14}$ (model) $] \mathrm{MeV} / c^{2}$, $\Gamma\left(\Lambda_{c}(2880)^{+}\right)=\left[5.43_{-0.71}^{+0.77}\right.$ (stat) \pm 0.29 (syst) ${ }_{-0.00}^{+0.75}$ (model) $] \mathrm{MeV}$.

Near-threshold enhancement in the $D^{0} p$ amplitude was also studied. This enhancement was found to be consistent with a hypothesis of one being a resonant state with 
mass and width: $M\left(\Lambda_{c}(2860)^{+}\right)=\left[2856.1_{-1.7}^{+2.0}\right.$ (stat) \pm 0.5 (syst) ${ }_{-5.6}^{+1.1}$ (model) $] \mathrm{MeV} / c^{2}$, $\Gamma\left(\Lambda_{c}(2860)^{+}\right)=\left[67.6_{-8.1}^{+10.1}\right.$ (stat) \pm 1.4 (syst) ${ }_{-20.0}^{+5.9}$ (model) $] \mathrm{MeV}$, and quantum numbers $J^{P}=$ $3 / 2^{+}$with the parity measured relative to that of the $\Lambda_{c}(2880)^{+}$-baryon. Other quantum number possibilities were excluded with a significance of more than $6 \sigma$. The phase motion of the $3 / 2^{+}$component with respect to the non-resonant amplitudes was obtained in a modelindependent way and is consistent with resonant behaviour. The $\Lambda_{c}(2860)^{+}$state mass is consistent with recent predictions based on the non-relativistic heavy quark-light diquark model and from QCD sum rules in the HQET framework for an orbital $D$-wave $\Lambda_{c}$ excitation with quantum numbers of $3 / 2^{+}$.

In this analysis were also obtained first constraints on the spin and parity of the $\Lambda_{c}(2940)^{+}$state, and its mass and width were measured. The most probable spinparity assignment for $\Lambda_{c}(2940)^{+}$is $J^{P}=3 / 2^{-}$but other solutions with spins $1 / 2$ to $7 / 2$ cannot be excluded. The mass and width of the $\Lambda_{c}(2940)^{+}$were measured to be: $M\left(\Lambda_{c}(2940)^{+}\right)=\left[2944.8_{-2.5}^{+3.5}(\right.$ stat $) \pm 0.4(\text { syst })_{-4.6}^{+0.1}($ model $\left.)\right] \mathrm{MeV} / c^{2}, \Gamma\left(\Lambda_{c}(2940)^{+}\right)=$ $\left[27.7_{-6.0}^{+8.2}\right.$ (stat) \pm 0.9 (syst) ${ }_{-10.4}^{+5.2}$ (model) $] \mathrm{MeV}$. The $J^{P}=3 / 2^{-}$allotment for $\Lambda_{c}(2940)^{+}$state is consistent with its interpretations as a $D^{*} N$ molecule or a radial $2 P$ excitation.

\section{$5 \Xi_{c}$ Family}

Since all three quarks in $\Xi_{c}$ are different, a lot more possible configurations are allowed. They can be divided into states for which the wave function of the light diquark is antisymmetric in flavour (analogue of $\Lambda_{c}$ ) or symmetric in flavour (analogue of $\Sigma_{c}$ ). The two main states are the only members of the family that decay weakly, and their masses, lifetimes, and many of their decay modes were measured [3]. Up till recently though these branching fractions were quoted relative to reference modes, and for $\Xi_{c}^{0}, \Xi_{c}^{+}$there was no experimental information at all on the absolute branching fractions of those reference modes at all. Determining these branching fractions is highly important, experimental information is crucial to validate theoretical models as well as to constrain the model parameters.

Belle Collaboration have performed an analysis of $B^{-} \rightarrow \bar{\Lambda}_{c}^{-} \Xi_{c}^{0}$ inclusively with respect to the $\Xi_{c}^{0}$ decay using a hadronic $B$-tagging method based on a full reconstruction algorithm, and exclusively for $\Xi_{c}^{0}$ decays into $\Xi_{c}^{-} \pi^{+}, \Lambda K^{-} \pi^{+}$, and $p K^{-} K^{-} \pi^{+}$final states [10]. The first measurements of the absolute branching fractions was reported: $\mathcal{B}\left(\Xi_{c}^{0} \rightarrow \Xi^{-} \pi^{+}\right)=[1.80 \pm$ 0.50 (stat.) \pm 0.14 (syst.) $] \%, \mathcal{B}\left(\Xi_{c}^{0} \rightarrow \Lambda K^{-} \pi^{+}\right)=[1.17 \pm 0.37$ (stat.) \pm 0.09 (syst.) $] \%, \mathcal{B}\left(\Xi_{c}^{0} \rightarrow\right.$ $\left.p K^{-} K^{-} \pi^{+}\right)=[0.58 \pm 0.23$ (stat.) \pm 0.05 (syst.) $] \%$. The measured $\mathcal{B}\left(\Xi_{c}^{0} \rightarrow \Xi^{-} \pi^{+}\right.$) is in agreement with the theoretical predictions within uncertainties. Now one can combine this measured $\Xi_{c}^{0}$ branching fractions, e.g. that for $\Xi_{c}^{0} \rightarrow \Xi^{-} \pi^{+}$, with $\Xi_{c}^{0}$ branching fractions measured relative to $\Xi_{c}^{0} \rightarrow \Xi^{-} \pi^{+}$to get absolute $\Xi_{c}^{0}$ branching fractions for other modes.

A small while later Belle Collaboration reported the first measurements of the absolute branching fractions for the $\Xi_{c}^{+}$ground state as a result of an analysis of $\bar{B}^{0} \rightarrow \bar{\Lambda}_{c}^{-} \Xi_{c}^{+}$ inclusively using a hadronic $B$-tagging method based on a full reconstruction algorithm, and exclusively with $\Xi_{c}^{+}$decays into $\Xi^{-} \pi^{+} \pi^{+}$and $p K^{-} \pi^{+}$final states [11]. The results are: $\mathcal{B}\left(\Xi_{c}^{+} \rightarrow \Xi^{-} \pi^{+} \pi^{+}\right)=[2.86 \pm 1.21$ (stat.) \pm 0.38 (syst.) $] \%, \mathcal{B}\left(\Xi_{c}^{+} \rightarrow p K^{-} \pi^{+}\right)=$ $[0.45 \pm 0.21$ (stat.) \pm 0.07 (syst.) $] \%$. These are the first measurements of the absolute branching fractions $\mathcal{B}\left(\Xi_{c}^{+} \rightarrow \Xi^{-} \pi^{+} \pi^{+}\right)$and $\mathcal{B}\left(\Xi_{c}^{+} \rightarrow p K^{-} \pi^{+}\right)$. The measured $\mathcal{B}\left(\Xi_{c}^{+} \rightarrow \Xi^{-} \pi^{+} \pi^{+}\right)$ value within uncertainties is consistent with the theoretical prediction. The ratio $\mathcal{B}\left(\Xi_{c}^{+} \rightarrow\right.$ $\left.p K^{-} \pi^{+}\right) / \mathcal{B}\left(\Xi_{c}^{+} \rightarrow \Xi^{-} \pi^{+} \pi^{+}\right)$was measured to be $0.16 \pm 0.06$ (stat.) \pm 0.02 (syst.), which is consistent with world-average value of $0.21 \pm 0.04$ [3] within uncertainties. Once again the measured $\Xi_{c}^{+}$branching fractions, e.g. for $\Xi_{c}^{+} \rightarrow \Xi^{-} \pi^{+} \pi^{+}$, can be combined with $\Xi_{c}^{+}$branching fractions measured relative to $\Xi_{c}^{+} \rightarrow \Xi^{-} \pi^{+} \pi^{+}$to obtain other absolute $\Xi_{c}^{+}$branching fractions. 
Table 2. A brief summary of the excited states of the $\Omega_{c}^{0}$-baryons [3].

\begin{tabular}{|c|c|c|c|c|}
\hline State & Decay mode & Mass, MeV $/ c^{2}$ & Width, MeV & $J^{P}$ \\
\hline \hline$\Omega_{c}^{* 0}$ & $\Omega_{c}^{0} \gamma$ & $2765.9 \pm 2.0$ & & $\frac{3}{2}^{+}$ \\
\hline$\Omega_{c}(3000)^{0}$ & & $3000.4 \pm 0.4$ & $4.5 \pm 0.7$ & \\
$\Omega_{c}(3050)^{0}$ & & $3050.20 \pm 0.13$ & $<1.2 @ 95 \% \mathrm{CL}$ & \\
$\Omega_{c}(3065)^{0}$ & $\Xi_{c}^{+} K^{-}$ & $3065.5 \pm 0.3$ & $3.5 \pm 0.4$ & \\
$\Omega_{c}(3090)^{0}$ & & $3090.0 \pm 0.5$ & $8.7 \pm 1.3$ & \\
$\Omega_{c}(3120)^{0}$ & & $3119.1 \pm 1.0$ & $<2.6 @ 95 \% \mathrm{CL}$ & \\
\hline
\end{tabular}

The states of the $\Xi_{c}$ family exist in many angular momentum constituent quarks configurations, each in the form of an isospin pair. But an evidence for the isospin partner for $\Xi_{C}(2930)^{0}$ was not seen till 2018. The $\Xi_{c}(2930)^{0}$ was seen in $B^{-} \rightarrow \Lambda_{c}^{+} \bar{\Lambda}_{c}^{-} K^{-}$decays [12]. The Dalitz plot was clearly not flat and the $\Lambda_{c}^{+} K^{-}$projection was consistent with being a single resonance with the parameters given in [3]. However, given the small sample size, it was impossible to rule out other hypothesis (such as two overlapping $\Xi_{c}$ resonances or a complex interference pattern between $\Xi_{c}$ and charmonium resonances). Recently this analysis was repeated by the Belle collaboration [13], using 1.5 times larger statistics, which made it possible to observe $\Xi_{c}(2930)^{0}$ signal with greater significance and increase measurement precision. The measured mass and width of the $\Xi_{c}(2930)^{0}$ were found to be $\left[2928.9 \pm 3.0\right.$ (stat.) ${ }_{-12.0}^{+0.9}$ (syst.) $] \mathrm{MeV} / \mathrm{c}^{2}$ and $\left[19.5 \pm 8.4\right.$ (stat.) ${ }_{-7.9}^{+5.9}$ (syst.) $] \mathrm{MeV}$, respectively.

Since the isospin of the $\Xi_{c}$ state is always equal to $1 / 2$ and an evidence has been found for the neutral $\Xi_{c}(2930)^{0}$, it was natural to search for the charged $\Xi_{c}(2930)^{+}$state as the substructure in $\bar{B}^{0} \rightarrow \Lambda_{c}^{+} \bar{\Lambda}_{c}^{-} \bar{K}^{0}$ decays. Both BaBar and Belle Collaborations have previously studied this decay mode using data samples of $230 \times 10^{6}$ and $386 \times 10^{6} B \bar{B}$ pairs, and found signals of $1.4 \sigma$ and $6.6 \sigma$ significances, respectively $[12,14]$. But neither experiment looked for possible intermediate states such as in the $\Lambda_{c} K_{S}^{0}$ system. Finally an updated analysis of $\bar{B}^{0} \rightarrow \bar{K}^{0} \Lambda_{c}^{+} \bar{\Lambda}_{c}^{-}$was performed by Belle [15]. The $4.1 \sigma$ evidence of the charged charmed baryon state $\Xi_{c}(2930)^{+}$was found in the $\Lambda_{c} K_{S}^{0}$ mass spectrum. The measured mass and width are $M\left(\Xi_{c}(2930)^{+}\right)=[2942.3 \pm 4.4$ (stat.) \pm 1.5 (syst.) $] \mathrm{MeV} / c^{2}$ and $\Gamma\left(\Xi_{c}(2930)^{+}\right)=$ $[14.8 \pm 8.8$ (stat.) \pm 2.5 (syst.) $] \mathrm{MeV}$. The mass and width difference between neutral and charged $\Xi_{c}(2930)$ states is $\Delta M=\left[-13.4 \pm 5.3\right.$ (stat.) ${ }_{-12.1}^{+1.7}$ (syst.) $] \mathrm{MeV} / c^{2}$ and $\Delta \Gamma=[4.7 \pm$ 12.2 (stat.) ${ }_{-8.3}^{+6.4}$ (syst.)] MeV, respectively. Due to the limited statistics it was not possible to perform an angular analysis and to determine the spin-parity of the $\Xi_{c}(2930)^{+}$state.

\section{$6 \Omega_{c}$ Family}

The short list of known doubly strange charmed baryons is summarized in the table 2 . The $\Omega_{c}^{* 0}$ is too light to decay strongly and therefore only decays to $\Omega_{c}^{0} \gamma$. It was discovered by BaBar [16] and confirmed by Belle [17]. Both measured the mass difference $M\left(\Omega_{c}^{* 0}\right)-M\left(\Omega_{c}^{0}\right)$, and both results are in excellent agreement with each other as well as with most theoretical predictions.

Recently the LHCb collaboration conducted a study of the spectrum of $\Xi_{c}^{+} K^{-}$, observing five new narrow excited states of $\Omega_{c}^{0}$-baryons [18] and thus representing the first excited doubly strange charmed baryons decaying strongly. Observation of these states in an inclusive process through a two-body decay did not allow to determine their quantum numbers. Additional information can be obtained by studying the possible three-body decays or by 
reconstructing these states in the decays of heavy baryons. Four of these states $\left(\Omega_{c}(3000)\right.$, $\Omega_{c}(3050), \Omega_{c}(3065)$ and $\left.\Omega_{c}(3090)\right)$ were confirmed by Belle with very similar parameters, although there is no evidence found for $\Omega_{c}(3120)$ [19].

\section{$7 \Xi_{c c}$ Family}

Until recently sightings of $\Xi_{c c}$ states have been reported only at the SELEX experiment. SELEX claimed observation of $\Xi_{c c}^{+}$at a mass of $3519 \mathrm{MeV} / c^{2}$ in the $\Lambda_{c}^{+} K^{-} \pi^{+}$and $p D^{+} K^{-}$final states [20]. In each case the signature is a small signal on top of an even smaller background: 15.9 events above an estimated background of $6.1 \pm 0.5$ for the $\Lambda_{c}^{+} K^{-} \pi^{+}$channel, and 5.4 events above $1.6 \pm 0.4$ for the $p D^{+} K^{-}$channel. These observations were controversial, primarily because the lifetime and the production rate of $\Xi_{c c}$ at SELEX were quite far from the expected ones. The theoretical $\Xi_{c c}^{+}$lifetime expectation is approximately 200-250 fs across a number of models [21], compared to a reported upper limit of 33 fs. Even more surprisingly, after comparison of the relative yields of $\Lambda_{c}^{+}$and $\Xi_{c c}^{+}$and further correction for acceptance and additional decay modes, SELEX estimated that $20 \%$ of its sample of $1,630 \Lambda_{c}^{+}$came from $\Xi_{c c}^{+}$ decays (presumably with a contribution of similar order from $\Xi_{c c}^{++}$). This somewhat contradicts expectations as it is much more difficult to produce a baryon with more than one unit of flavour since two heavy quark-antiquark pairs need to be created within a narrow kinematic window so they can combine into a baryon.

FOCUS experiment, BaBar, Belle, and later LHCb Collaborations searched for $\Xi_{c c}$ trying to reproduce the observation published by SELEX [22]. All groups researched $\Lambda_{c}^{+} K^{-} \pi^{+}$along with many other final states. Noone found any signal. Moreover in each case the samples of used $\Lambda_{c}^{+}$events were much larger than one of SELEX: yields of 19k for FOCUS, 600k for BaBar, $840 \mathrm{k}$ for Belle, and $820 \mathrm{k}$ for LHCb. Nevertheless since the production environments differ quite drastically from that of SELEX the possibility should not be excluded that the doubly charmed baryon cross section is significantly higher with a hyperon in the initial state for reasons that are not theoretically understood.

There is one further fine point: in the $\Lambda_{c}^{+} K^{-} \pi^{+}$final state several of the excited $\Xi_{c}$ states, in particular the $\Xi_{c}(2970)$ and $\Xi_{c}(3077)$, were discovered. None of these states were reported by SELEX (although they were also not specifically excluded). This creates another problem: if the production cross section at SELEX for these excited $\Xi_{c}$ states were not lower than for $\Xi_{c c}$ they should have been seen clearly, so their non-observation raises questions about the $\Xi_{c c}$ signals; and vice versa if the cross section for the excited $\Xi_{c}$ is smaller than for $\Xi_{c c}$ the mechanism must be highly exotic since the $\Xi_{c}$ states are not only lighter than $\Xi_{c c}$ but also closer in flavour content to the initial-state $\Sigma^{-}$.

Recently LHCb collaboration performed a study of the $\Lambda_{c}^{+} K^{-} \pi^{+} \pi^{+}$spectrum, observing a structure consistent with originating from a weakly decaying particle, identified as the doubly charmed baryon $\Xi_{c c}^{++}[23]$. The mass of the structure was measured to be $[3621.40 \pm 0.72 \pm 0.27 \pm 0.14] \mathrm{MeV} / c^{2}$, where the last uncertainty is due to the limited knowledge of the $\Lambda_{c}^{+}$mass, and its width is consistent with experimental resolution. The $\Xi_{c c}^{++}$ lifetime was measured by LHCb in a separate study and was found to be $\left(256_{-22}^{+24} \pm 14\right) \mathrm{fs}$ [24]. While the reported state is consistent with most theoretical expectations for the $\Xi_{c c}^{++}$baryon, it is inconsistent with being an isospin partner to the $\Xi_{c c}^{+}$state reported by the SELEX collaboration.

\section{Summary}

Recently observed excited $\Omega_{c}$ and ground $\Xi_{c c}^{++}$states present a unique opportunity to test and further improve theoretical models, that predict properties of heavy hadrons. First measure- 
ments of the absolute branching fractions for the ground states of the $\Xi_{c}$-baryons is of both practical and theoretical interest.

It should be noted that no direct measurements of the $J^{P}$ of any of the excited strange charmed baryons are available. Constraints on the quantum numbers can be inferred only from the decay pattern.

\section{Acknowledgements}

This work was supported by the Russian Ministry of Science and Higher Education contract 14.W03.31.0026.

\section{References}

[1] M. Gell-Mann, Phys. Lett. 8, 214 (1964);

G. Zweig, CERN-TH-401 (1964);

G. Zweig, Developments in the Quark Theory of Hadrons 1, 22 (1980).

[2] E. Klempt and J.-M. Richard, Rev. Mod. Phys. 82, 1095 (2010).

[3] M. Tanabashi et al. (Particle Data Group), Phys. Rev. D 98, 030001 (2018).

[4] M. Artuso et al. (CLEO Collaboration), Phys. Rev. Lett.86, 4479 (2001).

[5] R. Mizuk et al. (Belle Collaboration), Phys. Rev. Lett. 98, 262001 (2007).

[6] A. Abdesselam et al. (Belle Collaboration), arXiv:1908.06235 [hep-ex] (2019).

[7] B. Aubert et al. (BaBar Collaboration), Phys. Rev. Lett. 98, 012001 (2007).

[8] N. Isgur and M.B. Wise, Phys. Rev. Lett. 66, 1130 (1991);

H.-Y. Cheng and C.-K. Chua, Phys. Rev. D 75, 014006 (2007).

[9] R. Aaij et al. (LHCb Collaboration), J. High Energ. Phys. 2017, 30 (2017).

[10] Y. B. Li, C. P. Shen et al. (Belle Collaboration), Phys. Rev. Lett. 122, 082001 (2019).

[11] Y. B. Li, C. P. Shen et al. (Belle Collaboration), Phys. Rev. D 100, 031101(R) (2019).

[12] B. Aubert et al. (BaBar Collaboration), Phys. Rev. D 77, 031101 (2008).

[13] Y. B. Li, C. P. Shen et al. (Belle Collaboration), Eur. Phys. J. C 78, 252 (2018).

[14] N. Gabyshev et al. (Belle Collaboration), Phys. Rev. Lett. 97, 202003 (2006).

[15] Y. B. Li, C. P. Shen et al. (Belle Collaboration), Eur. Phys. J. C 78, 928 (2018).

[16] B. Aubert et al. (BaBar Collaboration), Phys. Rev. Lett. 97, 232001 (2006).

[17] E. Solovieva, R. Chistov et al. (Belle Collaboration), Phys. Lett. B 672, 1 (2009).

[18] R. Aaij et al. (LHCb Collaboration), Phys. Rev. Lett. 118, 182001 (2017).

[19] J. Yelton et al. (Belle Collaboration), Phys. Rev. D 97, 051102 (2018).

[20] M. Mattson et al. (SELEX Collaboration), Phys. Rev. Lett. 89, 112001 (2002);

A. Ocherashvili et al. (SELEX Collaboration), Phys. Lett. B 628, 18-24 (2005).

[21] Ch.-H. Chang, T. Li, X.-Q. Li, and Y.-M. Wang, Commun. Theor. Phys. 49, 993-1000 (2008).

[22] S.P. Ratti et al. (FOCUS Collaboration), Nucl. Phys. Proc. Suppl. 115, 33-36 (2003);

B. Aubert et al. (BaBar Collaboration), Phys. Rev. D 74, 011103 (2006);

R. Chistov et al. (Belle Collaboration), Phys. Rev. Lett. 97, 162001 (2006);

R. Aaij et al. (LHCb Collaboration), J. High Energy Phys. 12, 090 (2013).

[23] R. Aaij et al. (LHCb Collaboration), Phys. Rev. Lett. 119, 112001 (2017).

[24] R. Aaij et al. (LHCb Collaboration), Phys. Rev. Lett. 121, 052002 (2018). 